\title{
Thermoelastic stress analysis of composite granular materials
}

\author{
by P. Jongchansitto***, X. Balandraud** ${ }^{*}$ I. Preechawuttipong* and M. Grédiac ${ }^{\star *}$ \\ * Department of Mechanical Engineering, Faculty of Engineering, Chiang Mai University, 239 Huay Kaew Rd., Muang \\ District, Chiang Mai 50200, Thailand, pawarut j@cmu.ac.th, itthichai@dome.eng.cmu.ac.th \\ ** Clermont Université, Institut Pascal, UMR CNRS/UBP/IFMA 6602, Campus des Cézeaux, BP 80026, 63171 Aubière \\ cedex, France, balandraud@ifma.fr, michel.grediac@univ-bpclermont.fr
}

\begin{abstract}
The study deals with the stress distribution in two-dimensional "composite" granular media, i.e. built with cylinders made of different materials. Several non-cohesive granular materials were prepared from cylinders in polyoxymethylene (POM) and high-density polyethylene (HDPE). After compaction to reach static configuration, infrared thermography was applied to measure the temperature changes under cyclic loading in confined compression. Stress paths were then revealed by thermoelastic stress analysis. Results are analyzed in terms of statistical distribution of the stress level in the granular material.
\end{abstract}

\section{Introduction}

Granular materials are composed of particles whose macroscopic mechanical behavior is governed by the interactions between particles. Many examples of granular materials can be found in everyday life, such as soil, cereal, sugar, pharmaceutical product, snow, dust etc. They are constituted of grains or powders. The macroscopic mechanical behavior of granular media is generally complex. For this reason, the analysis of the mechanical response at the grain network scale is of great interest to understand the behavior of granular materials at the "continuum" macroscopic scale.

In former works, some full-field measurement techniques have been employed to study the mechanical behavior of such media. Most of them are two-dimensional (2D). The heterogeneity of contact forces was first observed in 1957 using 2D photoelastic particles which have the property to exhibit birefringence under stress [1]. Photoelasticimetry was then used with a specific image processing to obtain normal and tangential force components for each contact [2]. Digital image correlation was also used to measure displacement fields [3] and then strain fields [4] in 2D granular materials to analyze the internal structures of deformation. The use of $x$-ray tomography combined with three-dimensional digital image correlation was used to monitor the granular structure and the localized deformation at grain scale [5]. The Influence of boundary conditions and material properties on the development of shear band system was investigated by $x$-ray technique and particle image velocimetry (PIV) [6]. Strain components were also measured using particle image velocimetry [7].

Infrared (IR) thermography can be used for measuring stresses at the surface of a specimen subjected to a cyclic mechanical loading. Indeed, thermoelastic coupling predicts that temperature changes if elastic strain changes. Under cyclic loading at sufficiently high frequency, the temperature change in an isotropic material is directly proportional to the sum of the principal stresses of the stress tensor. This is the basis of the thermoelastic stress analysis [8], which has been used in various studies devoted to the mechanical characterization of materials and structures (see Refs. [912] for instance). Except for analyzing the heat transfer in granular materials [13], it seems that IR thermography was not employed to study the mechanical behavior of these materials.

The present study deals with IR measurements in 2D "composite" granular media, i.e. composed of cylinders made in different materials. The objective is to deduce the stress paths from the temperature variations under cyclic loading and then to perform a statistical analysis of the stress levels reached in the cylinders. Several non-cohesive configurations were prepared. They were different in terms of diameters used for the cylinders and numbers of cylinders for each type of material. After compaction, an IR camera captured the temperature variations due to the thermoelastic coupling during confined compressive cyclic loading. The stress levels in the cylinders were then deduced and analyzed.

\section{Experimental setup, measurement, and post-processing}

2D composite granular samples were prepared using two constitutive materials: polyoxymethylene (POM) and high-density polyethylene (HDPE). $60 \mathrm{~mm}$ in length cylinders were used. Different diameters were considered. Cylinders were randomly placed in a metallic rectangular frame. Granular samples were then subjected to vertical compression in confined configuration using a uniaxial testing machine. Note that the samples were non-cohesive. It means that no tensile contact forces are expected. Figure 1 presents the experimental setup: POM and HDPE cylinders are in black and white, respectively. The different tested configurations are given in Table 1 . They differ in terms of ratio between diameters and number of cylinders. The measurement of the contact stiffnesses between cylinders was preliminary 
performed with compression tests along a generatrix. The contact stiffness of POM cylinders is about four times higher than the contact stiffness of HDPE cylinders : $27.4 \mathrm{kN} / \mathrm{mm}$ and $6.4 \mathrm{kN} / \mathrm{mm}$, respectively.

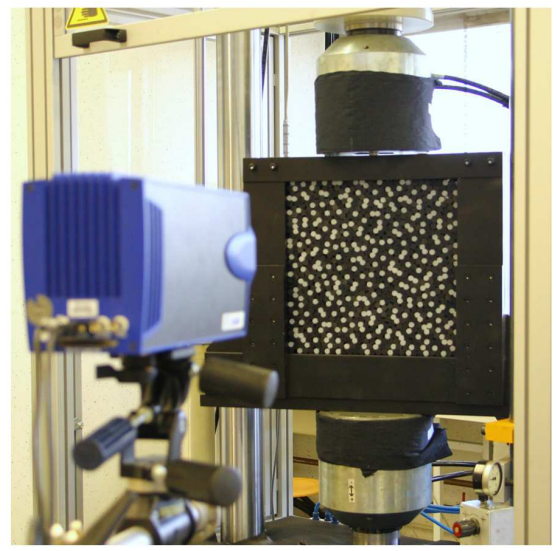

a)

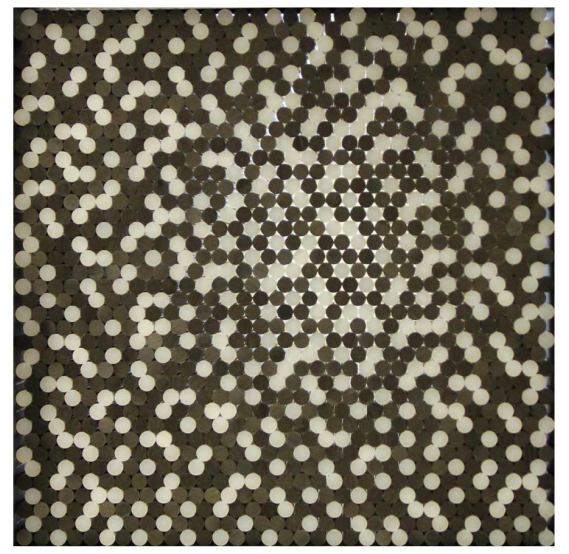

c)

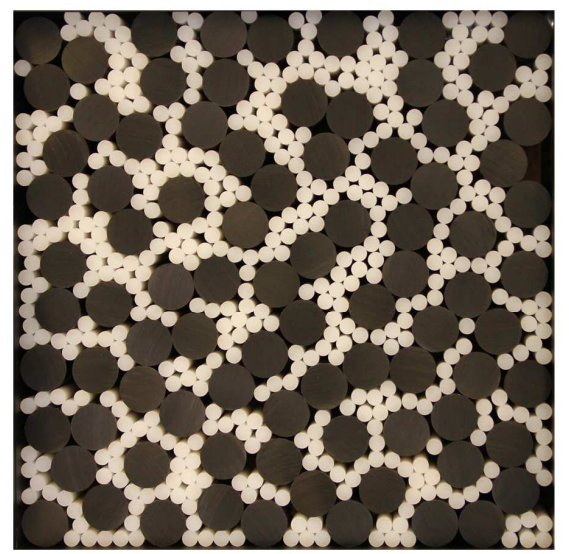

e)

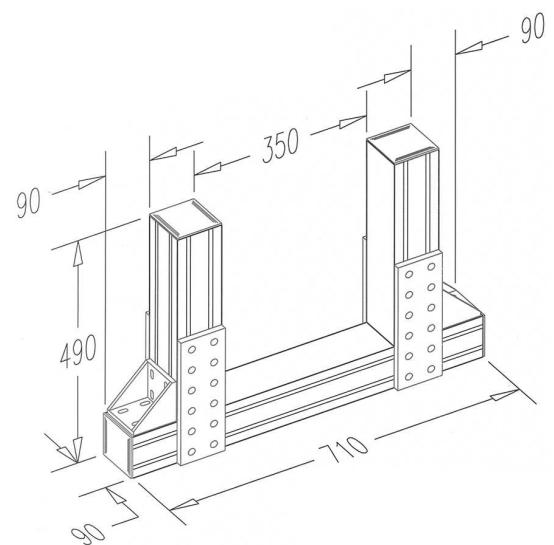

b)

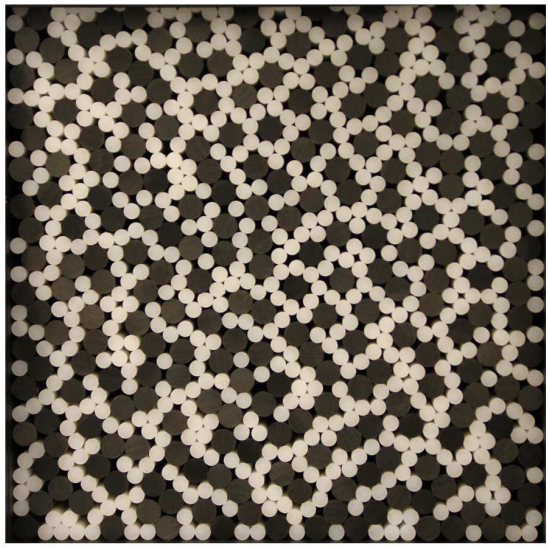

d)

Fig. 1. a) Picture of the experimental setup. Two reinforcement plates were added at the top the structure to ensure the rigidity of the frame. These plates prevent any bending of the two lateral parts of the frame under loading;

b) Frame geometry (dimensions in $\mathrm{mm}$ ); $\mathrm{c}-\mathrm{d}-\mathrm{e}$ ) Pictures of granular samples: configurations $A, B$ and $C$

Cyclic compressive tests were performed at ambient temperature with a uniaxial $\pm 250 \mathrm{kN}$ Schenck testing machine. Loading was force-controlled with a frequency of $3 \mathrm{~Hz}$, a force ratio of 0.1 and a maximum compression force of $-60 \mathrm{kN}$. In order to reach the static equilibrium configuration, the granular sample was first compacted. A Cedip Jade III-MWIR camera was used to capture the temperature fields on the front face of the cylinder network during the cyclic loading. The integration time and the acquisition frequency were set to $1500 \mu \mathrm{s}$ and $148 \mathrm{~Hz}$, respectively. The pixel size 
on the specimen was equal to $1.60 \mathrm{~mm}$. In the framework of a thermoelastic stress analysis, the relationship between $\Delta T$ and the amplitude of the sum of principal stresses $\Delta \sigma$ during cyclic loading is given by:

$$
\Delta T=A \Delta \sigma
$$

Note that cross-sections of cylinders were not painted in black. This enabled us to distinguish POM and HDPE from optical pictures in order to find the centers and diameters of cylinders to be used in the simulations. As thermal emissivity was not guaranteed close to one, coefficient $A$ was preliminarily identified from uniaxial tensile tests on non-painted rectangular sheets of the two materials: $0.0150 \mathrm{~K} / \mathrm{MPa}$ for POM and $0.0258 \mathrm{~K} / \mathrm{MPa}$ for HDPE. The amplitude $\Delta T$ of the temperature oscillations was calculated at each pixel on the specimen surface by Fourier analysis. As expected, experimental measurements exhibited temperature oscillations at the same frequency as the load. Let us denote by $\Delta T(x, y)$ the field of temperature amplitude, where $x$ and $y$ are the coordinates of the pixels in the cross section of the cylinder network. The value of $\Delta \sigma$ at the center of each cylinder is expected to be proportional to the sum of the amplitudes of the (compression) normal contact forces applied to the cylinder.

Table 1. Tested configurations

\begin{tabular}{ccccc}
\hline Configuration & $\begin{array}{c}\text { Ratio of diameters } \\
\text { DPOM / D }\end{array}$ & $\begin{array}{c}\text { Number of cylinders } \\
\text { POM : HDPE }\end{array}$ & $\begin{array}{c}\text { Ratio of cylinder numbers } \\
\text { POM : HDPE }\end{array}$ & $\begin{array}{c}\text { Total number } \\
\text { of cylinders }\end{array}$ \\
\hline A & 1 & $799: 436$ & $1: 0.55$ & 1235 \\
B & 1.6 & $239: 608$ & $1: 2.54$ & 847 \\
C & 3 & $86: 466$ & $1: 5.42$ & 552 \\
\hline
\end{tabular}

\section{Numerical simulations}

Simulations were performed for comparison purposes with experimental results. The molecular dynamics (MD) method was used. It is a well-known discrete element method. It has been widely used for the numerical simulation of granular materials since 1979, from the pioneering work of Cundall and Strack [14]. This method is based on an explicit algorithm in which the particles are considered as rigid bodies with non-conforming surface: the deformation of the particles is assumed to be very small and perfectly elastic [15]. The method is based on a predictor-corrector scheme with Gear's set of corrector coefficients for integration of the Newton's equations of motion [16]. The Coulomb's law of friction is a non-smooth relation. So a regularized Coulomb's law of friction [17] is considered because the integration of the equation of motion requires a smooth (mono-valued) law such that the friction force can be expressed as a monovalued function of the sliding velocity.

In practice, MD calculations are performed by the following scheme. Step 1: get initial conditions at time $t$, such as initial positions, velocities, time derivatives, number of time steps (iterations), number of particles, etc. Step 2: predict the positions, velocities and accelerations at time $t+\Delta t$, using the values at time $t$. Step 3: calculate contact forces between particles, and then obtain the new accelerations. Next, estimate the modified accelerations (amplitude of error between predicted and new accelerations). Step 4: correct the predicted positions, velocities, accelerations, etc. by using the modified accelerations obtained in step 3. Step 5: repeat steps 2-3 until the system reaches static equilibrium.

\section{Results and analysis}

The stress distribution in the tested monodisperse granular sample (configuration A, which is composed of cylinders with the same diameter) is shown in Figure 2-a. The color in each cylinder corresponds to the mean stress in the cylinder. Due to the monodisperse nature, all cylinders have here six neighbors, except the cylinders which are located at the boundary. It is noteworthy that high stress values exist along the boundary. "Triangular" contact patterns are revealed in the figure: they consist of inclined straight lines which are symmetrical with respect to the vertical load direction. This is typical of contact networks found in monodisperse granular materials. However, it can be noted that the stress field is heterogeneous owing to two types of materials (POM and HDPE) which have different stiffnesses. The other two configurations ( $B$ and $C$ ) exhibit stress networks which are more complex than that obtained for configuration $\mathrm{A}$. In particular, higher stress levels are observed in configuration $\mathrm{C}$.

Figure 2-b shows the contact force network obtained with the MD simulation for configuration A. Contact forces are represented by red lines. The thickness of the lines is proportional to the normal force amplitude. It can be observed that the force network consists of inclined straight lines, as observed in the experiment. Simulations and experiments are in global agreement. However, the high stresses which are experimentally found along the four boundaries are not obtained in the simulation. 


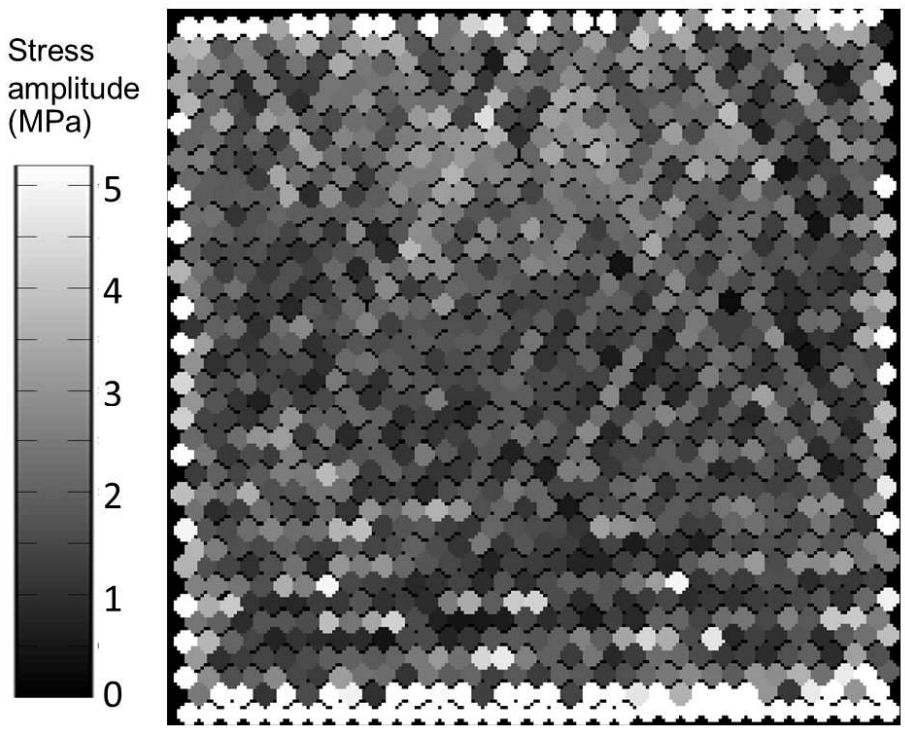

a)

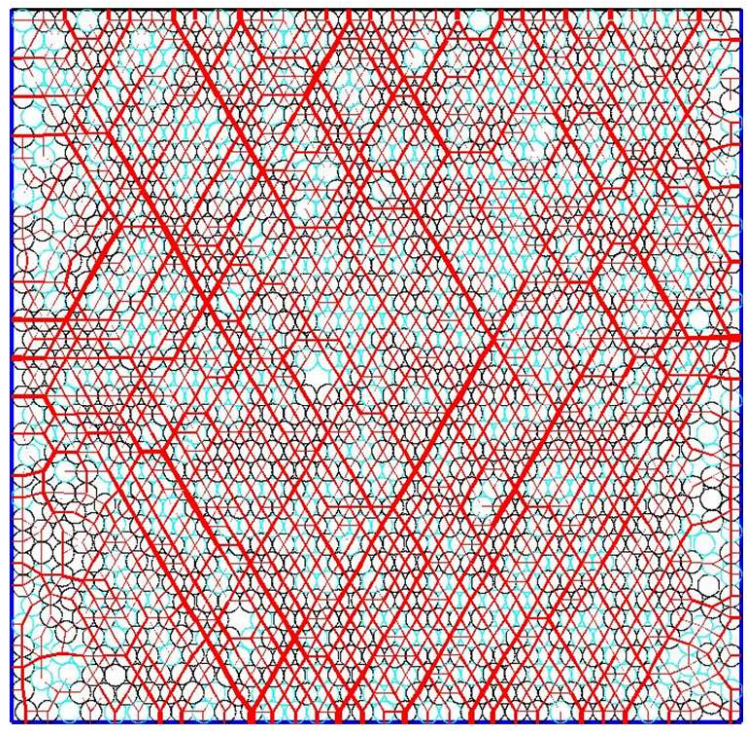

b)

Fig. 2. a) Stress field for configuration $A$. The color scale was yielded in order to remove the cylinders along the four boundaries from the analysis. b) Contact force network obtained from molecular dynamics simulation for configuration $A$. The thickness of the red lines is proportional to the force amplitude

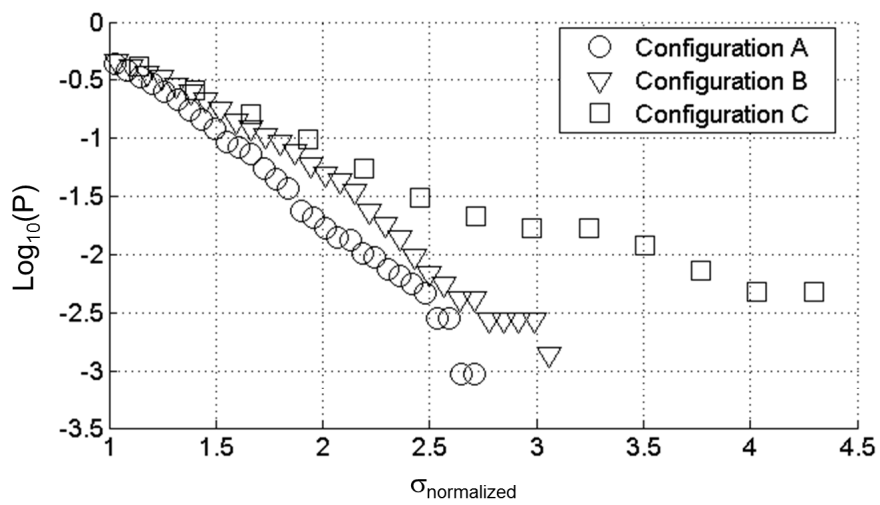

a)

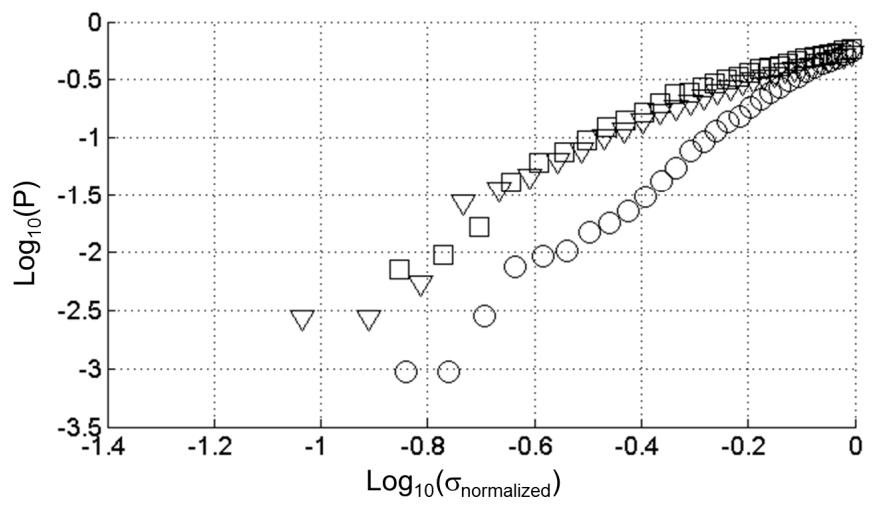

b)

Fig. 3. Stress distributions for the three configurations. a) Semi-logarithmic plot of the probability distributions of stresses which are greater than the mean value. b) Logarithmic plot of the probability distributions of stresses which are lower than the mean value 
A statistical data processing is employed to analyze the experimental results. Boundary cylinders are removed from the analysis. Table 2 presents the results in terms of mean stress over the whole granular material. Logically, for a same macroscopic load, the higher the percentage of void, the higher the mean stress. Figure 3 presents the distribution of the stresses in the granular materials. In this figure, stresses are normalized with respect to the mean value. For the three samples, about $40 \%$ of the cylinders have a stress level which is greater than the mean value: these cylinders are the so-called "strong network". The distribution law of the stresses in these cylinders follows an exponential decay: see Figure 3-a. In the cylinders which have a stress lower than the mean value ("weak network"), a power-law distribution is observed: see Figure 3-b. This property was obtained for the three tested specimens. It is in agreement with the simulations performed by molecular dynamics method. It can be noted that this property is well known from the numerical studies on contact forces [18,19]: our results exhibit this bimodal character which is usually observed in non-cohesive granular media made of one type of constitutive material only. This property seems to be also achieved in terms of compression stresses in our non-cohesive composite granular materials.

Table 2. Experimental results by statistical analysis

\begin{tabular}{cccc}
\hline Configuration & $\begin{array}{c}\text { Percentage of } \\
\text { void }\end{array}$ & $\begin{array}{c}\text { Mean stress over the whole } \\
\text { granular material }\end{array}$ & $\begin{array}{c}\text { Percentage of cylinders with a } \\
\text { stress greater than the mean value }\end{array}$ \\
\hline A & $11.9 \%$ & $1.91 \mathrm{MPa}$ & $44.2 \%$ \\
B & $16.9 \%$ & $2.89 \mathrm{MPa}$ & $46.3 \%$ \\
C & $15.3 \%$ & $2.67 \mathrm{MPa}$ & $41.2 \%$ \\
\hline
\end{tabular}

\section{Conclusion}

The present study is aimed at experimentally evidencing stress networks in 2D composite granular materials under confined compression, using infrared thermography. Three granular samples were prepared with cylinders made of two different constitutive materials exhibiting different stiffnesses. A thermoelastic stress analysis (TSA) was performed. This method enabled us to reveal the stress network under mechanical loading. It can be noted that the TSA method is a new experimental technique for granular materials to the best knowledge of the authors. It is of course complementary to other full-field measurement techniques such as photoelasticimetry, digital image correlation, etc., which have been already employed for such media. The technique is also complementary to simulations carried out within the framework of statistical analysis, as it was initiated in the present study. In particular, the bimodal character of non-cohesive granular materials [18] was found in the present study. The distribution law of stress was also the same as the distribution law found for contact forces [19].

\section{ACKNOWLEDGEMENTS}

The authors gratefully acknowledge the Thailand Research Fund through the Royal Golden Jubilee Ph.D. Program (Grant No. PHD/0159/2552) and the French Embassy in Thailand for their support during this research.

\section{REFERENCES}

[1] Dantu P., "Contribution à l'étude mécanique et géométrique des milieux pulvérulents", Proceedings of the 4th Int Conf on Soil Mechanics and Foundation Engineering, tome 1, Butterworth, London, pp. 144-148, 1957.

[2] Majmudar T.S. and Behringer R.P., "Contact force measurements and stress-induced anisotropy in granular materials", Nature 435, pp. 1079-1082, 2005.

[3] Richefeu V., Combe G., and Viggiani G., "An experimental assessment of displacement fluctuations in a 2D granular material subjected to shear", Geotechnique Letters 2, pp. 113-118, 2012.

[4] Hall S.A., Wood D.M., Ibraim E., and Viggiani G., "Localised deformation patterning in 2D granular materials revealed by digital image correlation", Granular Matter 12, pp. 1-14, 2010.

[5] Hall S.A., Bornert M., Desrues J., Pannier Y., Lenoir R., Viggiani G., and Bésuelle P., "Discrete and continuum analysis of localised deformation in sand using X-ray $\mu \mathrm{CT}$ and volumetric digital image correlation", Geotechnique 60, pp. 315-322, 2010.

[6] Wolf H., König D., and Triantafyllidis T., "Experimental investigation of shear band patterns in granular material", Journal of Structural Geology 25, pp. 1229-1240, 2003.

[7] Słomiński C., Niedostatkiewicz M., and Jacek T., "Deformation measurements in granular bodies using a particle image velocimetry technique", Archive of Hydro-Engineering and Environmental Mechanics 53, pp. 7194, 2006. 
[8] Dulieu-Barton J.M. and Stanley P., "Development and application of thermoelastic stress analysis", Journal of Strain Analysis for Engineering Design 33, pp. 93-104, 1998.

[9] Barone S. and Patterson E.A., "Polymer coating as a strain witness in thermoelasticity", Journal of Strain Analysis for Engineering Design 33(3), pp. 223-232, 1998.

[10] Emery T.R., Dulieu-Barton J.M., Earl J.S. and Cunningham P.R., "A generalised approach to the calibration of orthotropic materials for thermoelastic stress analysis", Compos Sci Technol 68(3-4), pp. 743-752, 2008

[11] Boyd S.W., Dulieu-Barton J.M. and Rumsey L., "Stress analysis of finger joints in pultruted GRP materials", Int. J. Adhes. Adhes. 26(7), pp. 498-510, 2006

[12] Moutrille M.P., Balandraud X., Grédiac M., Derrien K. and Baptiste D., "Applying thermoelasticity to study stress relief and crack propagation in aluminium specimens patched with composite material", Journal of Strain Analysis for Engineering Design 43(6), pp. 423- 434, 2008

[13] Sepulveda F. and Fudym O., "Infrared tracking from morphological image processing tools - Application to heat transfer characterization in granular media", Heat transfer engineering 32(9), pp. 787-799, 2011

[14] Cundall P.A. and Strack O.D.L., "A discrete numerical model for granular assemblies", Géotechnique 29(1), pp. 47-65, 1979

[15] Johnson K.L., "Contact Mechanics", Cambridge University Press, 1985

[16] Allen M.P. and Tildesley D.J., "Computer Simulation of Liquid”, Oxford Science Publications, 1987

[17] Preechawuttipong I., Peyroux R., Radjai F., and Rangsri W., "Static states of cohesive granular media", Journal of Mechanical Science and Technology 21(12), pp. 1957-1963, 2007

[18] Radjaï F., Wolf D.E., Jean M. and Moreau J.J., "Bimodal character of stress transmission in granular packings", Physical Review Letters 80(1), pp. 61-64, 1998

[19] Radjaï F., Jean M., Moreau J.J. and Roux S., "Force distributions in dense two-dimensional granular systems", Physical Review Letters 77, pp. 274-277, 1996 